\title{
Burden of malaria in mobile populations in the Greater Accra region, Ghana: a cross- sectional study
}

Nouhoum Diallo ${ }^{1,2}$, Patricia Akweongo ${ }^{1}$, Ernest Maya ${ }^{1}$, Moses Aikins ${ }^{1}$ and Bismark Sarfo ${ }^{\text {* }}$

\begin{abstract}
Background: The burden of malaria in mobile populations remains poorly documented in sub-Saharan Africa. This study determined the prevalence of malaria among hawkers and long-distance truck drivers in the Greater Accra region of Ghana.

Methods: A cross-sectional design using consecutive sampling method between June and July 2016 in Accra and Tema in Ghana was used in this study. The study population was hawkers who roam and sleep in the Market Streets, and long-distance truck drivers. Participants completed closed ended interview questionnaires on socio-demographic characteristics, primary residence and knowledge about malaria. Rapid diagnostic test and thick blood smears of each participant were stained with Giemsa and read using microscopy. Geographical position system (GPS) was used to collect the station locations of these mobile populations.

Result: The overall prevalence of malaria was $15.1 \%$ and Plasmodium falciparum was responsible for all malaria infection. The malaria prevalence was 18.9 and $10.9 \%$ respectively among hawkers and truck drivers $(p<0.05)$. The hawkers, the single and the no formal educated participants were more likely to get malaria than the long-distance truck drivers $(\mathrm{OR}=1.91,95 \% \mathrm{Cl} 1.07-3.42)$, the married $(\mathrm{OR}=1.9495 \% \mathrm{Cl} 1.11-3.40)$ and the educated participants $(\mathrm{OR}=2.5695 \% \mathrm{Cl} 1.10-5.93)$, respectively. After controlling for other variables, marital status $(\mathrm{OR}=2.6095 \% \mathrm{Cl} 1.43-$ 4.73) and educational level $(\mathrm{OR}=2.7095 \% \mathrm{Cl} 1.08-6.77)$ were statistically significantly associated with malaria.

Conclusion: This study demonstrated that the prevalence of malaria is high among hawkers and long distance truck drivers. Sociodemographic characteristics, such as marital status, occupation and educational level are significantly associated with malaria. The station locations as determined by GPS technology will make these mobile populations easier to reach for any malaria intervention.
\end{abstract}

\section{Background}

Plasmodium falciparum is the most important threat to public health at a global scale, responsible for more than $90 \%$ of the World's malaria mortality [1]. An estimated 212 million new cases of malaria occurred worldwide in 2015 (range: 148-304 million) and the majority of the cases occurred in the African Region (90\%). [2]. According to a World Health Organization (WHO) report, malaria is responsible for approximately 1800 admissions at health care system and 10 deaths for every 100,000

\footnotetext{
*Correspondence: bsarfo@ug.edu.gh

1 School of Public Health, University of Ghana, Legon-Accra, Accra, Ghana Full list of author information is available at the end of the article
}

population in Ghana [3]. The movement of human populations has been described as a major challenge tackling malaria control and elimination programmes since they require a knowledge of how the spatial distribution of malaria shifts through time and across multiple locations that become interconnected through population displacements [4]. Mobile populations from higher transmission settings risks reintroduction and resurgence in malaria-free areas, and has compromised elimination efforts in the past years $[5,6]$. In non-elimination areas, understanding the dynamics of parasite movements from local hotspots of transmission is crucial for the development of appropriate surveillance and response system by 
identifying both the regions where imported infections originate and how they may contribute substantially to transmission [7].

Ghana and many sub-Saharan African countries have been experiencing rapid population growth and urbanization with high rates of migration into the cities and large towns, creating unemployment and overcrowding [8]. Most people without accommodation move around in the cities, do casual work and sleep wherever they find space during the night. Mobile populations, such as hawkers and long-distance truck drivers are all among the malaria at-risk populations who do not benefit from protection against mosquitoes, such as long-lasting, insecticide-treated bed nets (LLIN). Different features make migrants an at-risk of malaria population, including their socio-cultural context, economic activities, accessibility to health services, access to anti-malarials, service availability and readiness, behavioural parameters, language barriers, emergence of drugresistant strains, activities for social mobilization, ecology, access to safe water and sanitation [9].

These groups tend to carry malaria parasites and could be a major source of residual transmission that could thwart the effort of any control strategy. Understanding malaria transmission in these mobile populations will be crucial in developing targeted interventions by the National Malaria Control Programmes [10].

In the literature, intra-country mobility and malaria risk remain poorly documented in sub-Saharan Africa. The magnitude of malaria in mobile populations and its contribution to the local malaria situation in Ghana is unknown. This study assessed the burden of malaria in mobile populations and their malaria-related risk factors in the Greater Accra Region of Ghana. The outcome of this study provides very useful information that will bridge the knowledge gap for programmatic improvement of malaria control in Ghana and the sub-region.

\section{Methods}

\section{Study area}

The study was conducted in two cities, Accra and Tema in the Greater Accra Region, one of the country's ten administrative regions. It covers an area of about $420 \mathrm{~km}^{2}$ [11], with an estimated population of 4010,054 by the Statistical Service census of 2010. In 2010, malaria positive test results from Accra Metropolis was 8\% [12]. Madina is a particularly interesting setting to examine the relationship between migration and malaria prevalence. It has a large migrant community. Madina is a suburb of Accra, the capital of Ghana, and in the LA-NKwantanang-Madina Municipal Assembly, in the East district of the Greater Accra Region. It lies about seventeen kilometres to the northeast of Accra, about four kilometres beyond the University of Ghana campus.
Tema is located in the Greater Accra Region with an estimated population of 402,637, which accounts for 392,044 people from urban areas and 10,593 from rural areas according to the Ghana Statistical Service census of 2010. According to the Ghana Health Service, District Health Information Management System data from January to October 2012, the percentage of malaria positive tests was about $12 \%$ in Tema. Tema has a port, which is the bigger of the two seaports of Ghana, handling $80 \%$ of the country's national exports and imports. The harbour is situated along the Gulf of Guinea, 18 miles from the capital Accra, and it serves both as a loading and unloading port for goods, both for Ghana and the land-locked countries to the north, such as Burkina Faso, Mali and Niger. Long-distance truck drivers are present in this city, and they convey goods from the harbour to other parts of Ghana, and neighbouring countries.

\section{Study population (mobile population)}

Mobile populations are defined as non-residents of the study area who had travelled there within the previous weeks and without accommodation. Most of the hawkers had travelled from the Northern part of Ghana to Accra the capital city in search of job opportunities as head porters. They often move around the market areas and assist shoppers in carrying their goods to their vehicles for a fee.

The truck drivers in this study are those who use their trucks in carrying goods from Tema harbour to the Northern part of Ghana as well as those who carry goods to the Economic Community of West African States (ECOWAS) member countries such as Mali, Burkina Faso and Niger.

\section{Sampling procedure and data collection}

It was estimated that the prevalence of malaria among mobile population (long-distance truck drivers) in Niger Delta of Nigeria was 35\% [13]. Using the formula, $\mathrm{n}=\left(\mathrm{z}^{2} \mathrm{pq}\right) / \mathrm{d}^{2}$ with $\mathrm{n}=$ sample size, $\mathrm{p}=$ proportion of malaria in mobile population (35\%); $\mathrm{q}=1-\mathrm{p} ; \mathrm{z}=1.96$ and $d=5 \%$ at $95 \%$ confidence interval sample size of 350 study participants was estimated for this study. The study team compiled initial information from officials at Ghana Ports and Harbours Authority and the Queen mother of Madina Market Women Association, to identify locations and times in the study area that hawkers and long-distance truck drivers could be contacted. Mobile populations was defined as a non-resident of the study area who had travelled there within the previous weeks and without accommodation. Participants were recruited within each location using a consecutive sampling technique between June and July 2016. Volunteers were eligible if they were mobile populations (hawkers or long-distance truck 
drivers as defined above), aged 18 years or older, have the ability to provide informed consent and living in Madina market or Tema Harbour during the study period. Participants who consented were examined by a member of the study team who is a clinician. Subsequently, data on age, sex, education, knowledge of malaria transmission and prevention, and sleeping and mobility pattern were obtained through a closed ended questionnaire. Finger prick blood samples were also collected from participants to make thick smears, and examined for asexual parasites after staining with 5\% Giemsa. Blood smears were read within 1 week after collection, by two trained microscopists using WHO standard procedures. Malaria parasitaemia was defined by the presence of asexual form of Plasmodium by microscopic examination. Asymptomatic malaria was defined as absence of history of fever and symptomatic malaria as presence of history of fever in the past 2 weeks. Participants were also screened with malaria Rapid Diagnostic Test using CareStart ${ }^{\mathrm{TM}}$ Malaria (Histidine Rich Protein 2) HRP2(Pf) (Access Bio, INC, 65 Clyde Road Suite A Somerset NJ 08873 USA) recommended by the Ghana National Malaria Control Programme. Positive cases with Rapid Diagnostic Tests were managed with artemether $20 \mathrm{mg}+$ lumefantrine $120 \mathrm{mg}$. The location and distribution point of these mobile populations were collected using Geographical Position System technology (GPSMAP ${ }^{\circledR}$ 62, GARMIN, Kansas city, USA).

\section{Quality assurance and quality control}

Parasite density was estimated by counting the number of asexual parasites per 200 leukocytes and multiplying by 40 , assuming 8000 leukocytes $/ \mathrm{mL}$. Microscopists were kept blinded from the RDTs result until the smear parasite count was provided. Double entry for all data were reported.

\section{Data processing and analysis}

Data were double entered using Microsoft Excel. Statistical analyses were performed using Statistical Package Stata, version 13.0 (StataCorp LP. College Station, Texas, USA). Frequency and percentage were presented for categorical variables. Malaria test was categories into two: No malaria (absence of malaria parasites in the blood analysed by microscopy) and malaria (presence of malaria parasites in the blood analysed by microscopy). Chi square or fisher's exact test were used to assess the differences in sociodemographic and malaria knowledge factors between subjects. Subsequently, simple and multivariable logistic regression models were employed to analyse the risk factors of malaria. Both unadjusted and adjusted logistic regression models were performed. Odds ratios (OR) with $95 \%$ confidence intervals $(95 \%, \mathrm{CI})$ were presented. Two tailed $\mathrm{p}$ values $<0.05$ were considered statistically significant.

Data from the Geographical Position System (GPS) were managed with a software called Base camp, produced by Environmental System Research Institute (ESRI, Kansas City, USA). Base camp is specifically designed for Garmin GPS's. Data were first downloaded in Gpx format. Gpx is a format that Microsoft excel readily accepts for data or information analysis. The data could have easily been downloaded in "shapefile" format which is actually the format that would be required in the long run to make the analysis. Yet, the former was chosen to link each GPS point collected on the field to the exact questionnaire administered on the field of study. Since Gpx format readily accepts spreadsheet, it then makes it prudent and best to use that format to link both questionnaire information and GPS data.

After GPS data were collected and cleaned in the spreadsheet, a Geographical Information System (GIS) software was used for data management. Arcgis version 10.2.2 (ESRI, Kansas City, USA) was used in the data management procedure to generate the map showing sampling sites and malaria cases among the study participants.

\section{Ethical consideration}

All study procedures were clearly explained to participants while obtaining informed consent. Study participants were assured of the confidentiality, data safety and appropriate data usage. The study received approval from the Ghana Health Service Ethics Committee (GHS-ERC $72 / 02 / 16)$.

\section{Results}

\section{Sociodemographic characteristic of participants}

Overall, 390 people aged $18-70$ years old participated in the study. A total of 205 (52.56\%) participants were from Madina market and the rest 185 (47.44\%) from Tema Harbour. In all, 216 (55.38\%) were males, females were $174(44.62 \%)$ and $62.82 \%$ (245) were married. Participants were involved in two main occupation 206 (52.82\%) were hawkers and long-distance truck drivers were 184 (47.18\%). Regarding their educational status, 83 (21.28\%) obtained at least secondary school education, 81 (20.77\%) had primary school education and the majority 226 $(57.95 \%)$ had no formal education (Table 1 ).

\section{Malaria prevalence and malaria knowledge factors}

The prevalence of malaria in this study was $15.13 \%$ using microscopy and $15.38 \%$ with rapid diagnostic tests (Fig. 1). Plasmodium falciparum was responsible for all malaria infection observed in this study. Most participants had history of fever in the past 2 weeks. 
Table 1 Sociodemographic characteristics of participants

\begin{tabular}{lcc}
\hline Variable & Frequency $(\mathbf{N}=\mathbf{3 9 0})$ & Percentage (\%) \\
\hline Age, year & & \\
$<20$ & 72 & 18.46 \\
$20-29$ & 177 & 45.38 \\
$30-39$ & 71 & 18.21 \\
$40-70$ & 70 & 17.95 \\
Locality & & \\
Medina market & 205 & 52.56 \\
Tema harbour & 185 & 47.44 \\
Gender & & \\
Male & 216 & 55.38 \\
Female & 174 & 44.62 \\
Marital status & & \\
Single & 145 & 37.18 \\
Married & 245 & 62.82 \\
Occupation & & \\
Truck driver & 184 & 47.18 \\
Hawker & 206 & 52.82 \\
Educational level & & \\
No schooling & 226 & 21.97 .95 \\
Primary & 81 & \\
At least secondary & 83 & \\
\hline
\end{tabular}

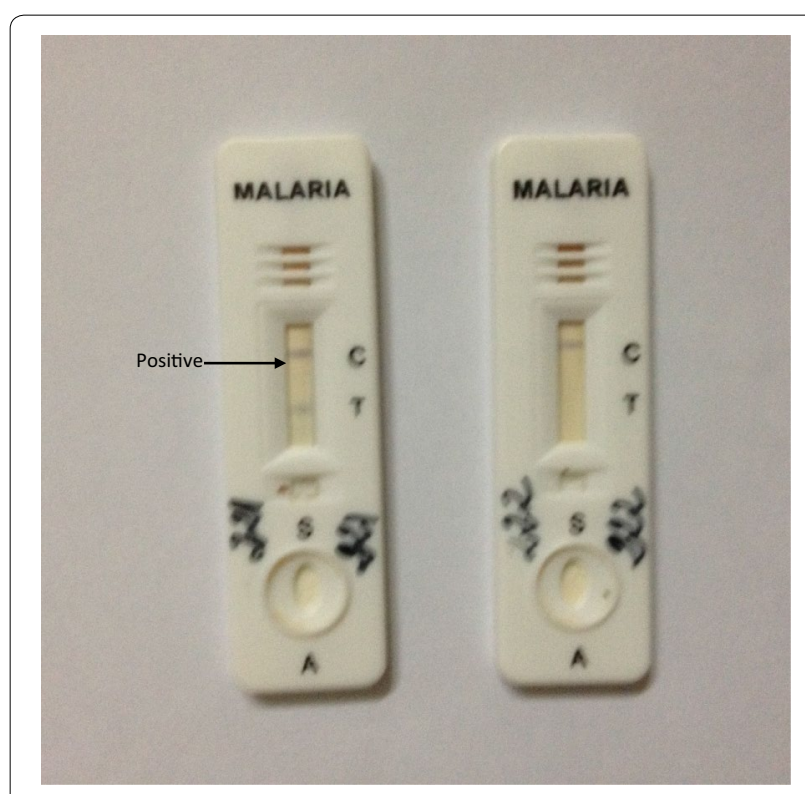

Fig. 1 Rapid diagnostic test (RDT) results: positive (221) and negative (222). C control and $T$ test result

360 (92.31\%) had no insecticide-treated bed net, while $30(7.69 \%)$ had a bed net. A total of 365 (93.59\%) knew about malaria, while 25 (6.41\%) said they didn't know. $85.90 \%$ (335) of the participants stated the correct mode of malaria transmission. Out of 390 participants, 332 (85.13\%) stated correct measures of preventing malaria, while 58 (14.87\%) said they did not know (Table 2).

\section{Sociodemographic characteristics associated with malaria and its prevalence}

The epidemiological characteristics of malaria and the sociodemographic characteristics are reported in Table 3. The Chi square or Fisher's exact test showed that the prevalence of malaria among participants had significant differences $(\mathrm{p}<0.05)$ in locality, marital status, occupation, educational level, while it had no significant difference $(p>0.05)$ in age groups and gender.

\section{Malaria knowledge factors associated with malaria and its prevalence}

The epidemiological characteristics of malaria and malaria knowledge factors are reported in Table 4. The Chi square or fisher's exact test showed that the prevalence of malaria among participants had no significant difference ( $p>0.05$ ) in history of fever in the last 2 weeks, have a bed net, know malaria, knowledge of malaria transmission and prevention methods.

\section{Factors associated with malaria from logistic regression}

All statistically significant variables in univariate analysis were applied to perform a multivariate logistic regression analysis, and the results are presented in Table 5 . The results showed that marital status, occupation and educational level were significantly associated with malaria. The hawkers, the single and the no formal educated participants were more likely to get malaria than the longdistance truck drivers $(\mathrm{OR}=1.91,95 \%$ CI 1.07-3.42), the married $(\mathrm{OR}=1.94,95 \% \mathrm{CI} 1.11-3.40)$ and the educated participants $(\mathrm{OR}=2.56$ 95\% CI 1.10-5.93), respectively. After controlling for occupation marital status $(\mathrm{OR}=2.60,95 \%$ CI 1.43-4.73) and educational level $(\mathrm{OR}=2.70,95 \%$ CI 1.08-6.77) were statistically significantly associated with malaria.

\section{Localizations of the halting point of this mobile populations using GPS technology}

The maps in Figs. 2 and 3 show the distribution of positive and negative malaria cases determined by microscopy at the Madina market and Tema harbour, respectively. The insert pie chart provides a clear-cut and distinct representation of the different proportions of malaria cases in the study site.

\section{Discussion}

The microscopy remains the gold standard for malaria diagnosis. This study indicates that the prevalence of malaria was $15.1 \%$ in these mobile populations. This 
Table 2 Malaria prevalence and malaria knowledge factors

\begin{tabular}{lrl}
\hline Variable & Frequency $(\mathbf{N}=\mathbf{3 9 0})$ & Percentage (\%) \\
\hline Microscopy result & & \\
Negative & 331 & 84.87 \\
Positive & 59 & 15.13 \\
RDT result & \\
Negative & 330 & 84.62 \\
Positive & 60 & 15.38 \\
History of fever & & \\
No & 152 & 38.97 \\
Yes & 238 & 61.03 \\
Have a bed net & & \\
No & 360 & 92.31 \\
Yes & 30 & 7.69 \\
Know malaria & & \\
No & 25 & 6.41 \\
Yes & 365 & 93.59 \\
Malaria transmission & & \\
No & 55 & 85.90 \\
Yes & 335 & 14.87 \\
Prevention methods & & 85.13 \\
No & 58 & \\
Yes & 332 & \\
\hline
\end{tabular}

Table 3 Sociodemographic characteristics associated with malaria and its prevalence

\begin{tabular}{lrcc}
\hline Variable & No malaria & Malaria & p value \\
\hline Age, year (n, \%) & & & 0.055 \\
$<20$ & $57(79.17)$ & $15(20.83)$ & \\
$20-29$ & $148(83.62)$ & $29(16.38)$ & \\
$30-39$ & $60(84.51)$ & $11(15.49)$ & \\
$40-70$ & $66(94.29)$ & $4(5.71)$ & \\
Locality (n, \%) & & & $0.024^{*}$ \\
Accra & $166(80.98)$ & $39(19.02)$ & \\
Tema & $165(89.19)$ & $20(10.81)$ & \\
Gender (n, \%) & & & 0.296 \\
Male & $187(86.57)$ & $29(13.43)$ & \\
Female & $144(82.76)$ & $30(17.24)$ & \\
Marital status (n, \%) & & & $0.018^{*}$ \\
Single & $115(79.31)$ & $30(20.69)$ & \\
Married & $216(88.16)$ & $29(11.84)$ & \\
Occupation (n, \%) & & & $0.027^{*}$ \\
Truck driver & $164(89.13)$ & $20(10.87)$ & \\
Hawker & $167(81.07)$ & $39(18.93)$ & \\
Educational level (n, \%) & & & $0.019^{*}$ \\
No schooling & $182(80.53)$ & $44(19.47)$ & \\
Primary & $74(91.36)$ & $7(8.64)$ & \\
At least secondary & $75(90.36)$ & $8(9.64)$ & \\
\hline
\end{tabular}

No malaria absence of malaria parasites in the blood analysed, Malaria presence of malaria parasites in the blood analysed; * $p<0.05$

\begin{tabular}{lrrr}
$\begin{array}{l}\text { Table } 4 \text { Malaria } \\
\text { with malaria and its prevalence }\end{array}$ & factors & associated \\
\hline Variable & No malaria & Malaria & p value \\
\hline History of fever & $124(81.58)$ & $28(18.42)$ & 0.147 \\
No & $207(86.97)$ & $31(13.03)$ & \\
Yes & & & 0.065 \\
Have a bed net & $302(83.89)$ & $58(16.11)$ & \\
No & $29(96.67)$ & $1(3.33)$ & \\
Yes & $20(80.00)$ & $5(20.00)$ & \\
Know malaria & $311(85.21)$ & $54(14.79)$ & \\
No & & & 0.482 \\
Yes & $44(80.00)$ & $11(20.00)$ & \\
Malaria transmission & $287(85.67)$ & $48(14.33)$ & \\
No & & & 0.377 \\
Yes & $47(81.03)$ & $11(18.97)$ & \\
Prevention methods & $284(85.54)$ & $48(14.46)$ & \\
No &
\end{tabular}

No malaria absence of malaria parasites in the blood analysed, Malaria presence of malaria parasites in the blood analysed

Table 5 Factors associated with malaria from logistic regression

\begin{tabular}{|c|c|c|c|c|}
\hline Variable & $\mathrm{OR}^{\mathrm{a}}(95 \% \mathrm{Cl})$ & $p$ value & $\mathrm{OR}^{\mathrm{b}}(95 \% \mathrm{CI})$ & $\mathrm{p}$ value \\
\hline \multicolumn{5}{|l|}{ Marital status } \\
\hline Married & 1 & & 1 & \\
\hline Single & $1.94(1.11-3.40)$ & $0.020^{*}$ & $2.60(1.43-4.73)$ & $0.002^{* *}$ \\
\hline \multicolumn{5}{|l|}{ Occupation } \\
\hline Truck driver & 1 & & 1 & \\
\hline Hawker & $1.91(1.07-3.42)$ & $0.028^{*}$ & $1.74(0.90-3.36)$ & 0.099 \\
\hline \multicolumn{5}{|l|}{ Educational level } \\
\hline Primary & 1 & & 1 & \\
\hline No schooling & $2.56(1.10-5.93)$ & $0.029^{*}$ & $2.70(1.08-6.77)$ & $0.034^{*}$ \\
\hline $\begin{array}{l}\text { At least second- } \\
\text { ary }\end{array}$ & $1.13(0.39-3.27)$ & 0.825 & $1.20(0.40-3.52)$ & 0.741 \\
\hline \multicolumn{5}{|c|}{ OR Odds ratio, $95 \%$ Cl $95 \%$ confidence interval } \\
\hline \multicolumn{5}{|l|}{$* p<0.05$} \\
\hline \multicolumn{5}{|l|}{$* * p<0.01$} \\
\hline \multicolumn{5}{|l|}{ a Crude OR } \\
\hline b Adjusted for a & & & & \\
\hline
\end{tabular}

prevalence of malaria seems high in this adult populations from an area of malaria endemicity, where people develop immunity that protects them against high parasitaemia and clinical disease over a number of years [14]. However hawkers and long-distance truck drivers are among the malaria at-risk populations who do not benefit from some of the malaria intervention programs including the free distribution of LLINs that protect against mosquitoes bites and reduce malaria incidence [15]. The 
prevalence of malaria in our study is higher than a study conducted among migrant farm workers in Amhara region, in Ethiopia. The prevalence of malaria in this study using rapid diagnostic test was $12 \%$ of 605 participants [16]. The difference in the prevalence between the two studies could be explained by the proportion of participants who had access to LLIN. This proportion was 7.9\% in the Greater Accra region, in Ghana and 11.9\% in the Amhara region, in Ethiopia. This study prevalence was largely higher than an adult women clinical study conducted in Wakiso district of Uganda. This study found a prevalence of $2.3 \%$ among 86 participants [17]. The active case detection used in the study and the stable populations status of the non-pregnant adult women in Wakiso district, Uganda could justify this observed difference.

The study found a prevalence of 18.9 and $10.9 \%$, respectively among hawkers and long-distance truck drivers indicating higher prevalence among hawkers than truck drivers. Hawkers move around in the market, and sleep wherever they find space during the night. Long-distance truck drivers on the other hand are very mobile and travel through Ghana ten administrative regions and neighbouring countries. Most of the drivers had to frequently stay overnight and regularly sleep in their trucks to protect their loads. Most (92.3\%) of the study participants, had not used LLINs.

The study showed that asymptomatic malaria cases of $18.4 \%$ were higher than symptomatic cases of $13 \%$. In malaria endemic area, adult populations develop immunity that protects against high parasitaemia and clinical disease after some years of continue exposition to mosquito bites [14]. A study conducted on malaria parasitaemia among long-distance truck drivers in the Niger delta of Nigeria reported a prevalence of $35 \%$. The majority of the truck drivers studied were asymptomatic although some of them complained of regular fatigue, aches and weakness [13]. The reason for the high prevalence observed in the Nigeria study may be due to the fact that the study was carried out in an area of high incidence of malaria compare to Greater Accra region of Ghana. European visitors to the area described it as the "white

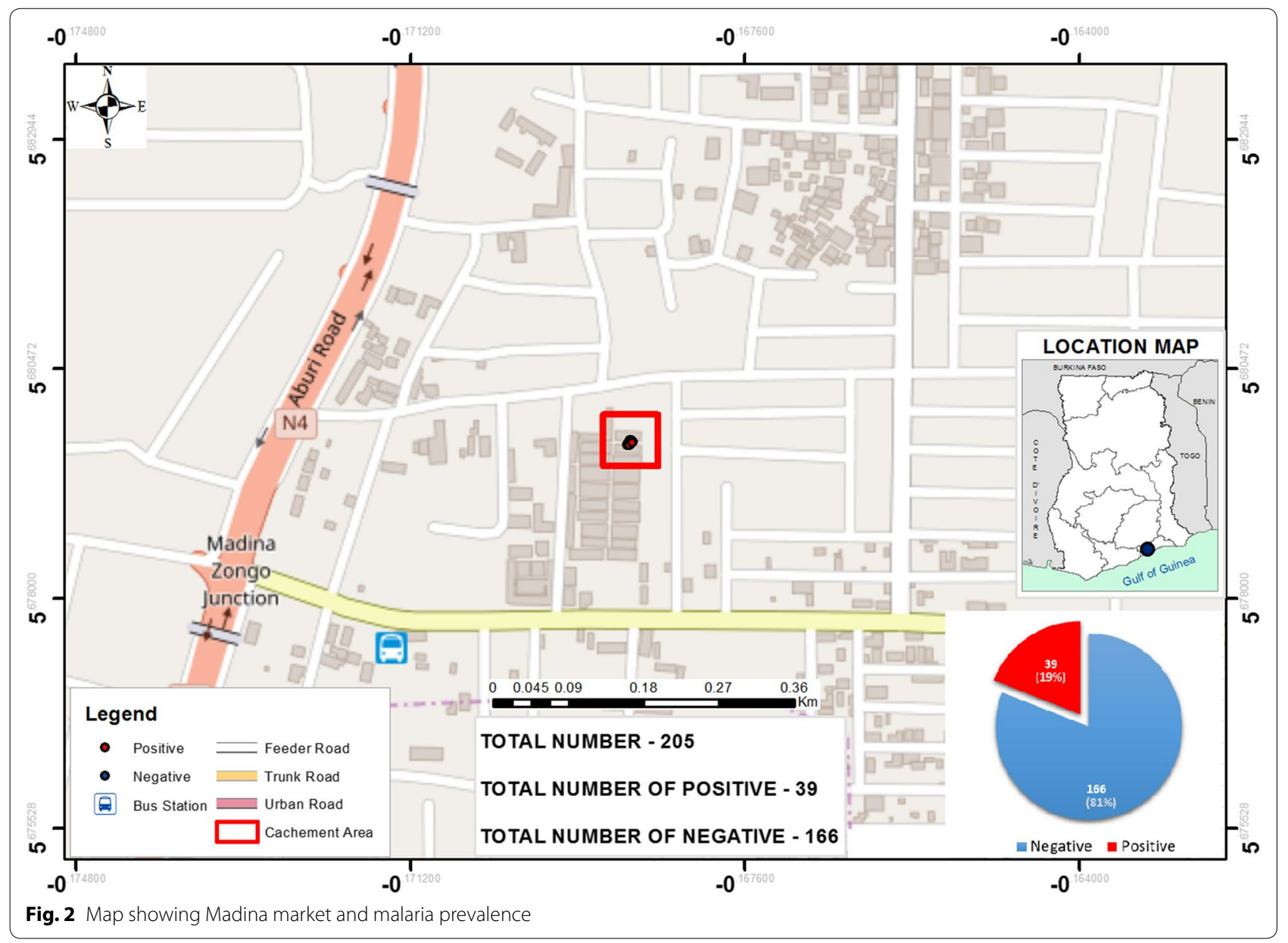


man's grave-yard" because of the high malaria related mortality rate they experienced [13].

The study showed that marital status, occupation and education level are risks factors strongly related to malaria. Those who are single had a higher odds of getting malaria than those who are married in this study. This may be due to the use of LLIN which is one of the strategies to reduce morbidity and mortality of malaria. The implementation of intervention that made LLIN available and free of charge especially for children and pregnant women could help married couple to be protected against mosquito bites. Marital status was a significant determinant of LLIN use in an urban area of Lagos state, Nigeria [18].

The study found that 'no formal educated' participants were more susceptible to malaria than 'formal educated' participants. This could be explained by the fact that those who have reached at least primary level of education might have been taught lessons on malaria in school, and are also more likely to read and comprehend malaria messages on tracts, radio or television. These findings were confirmed by others who reported that the knowledge of malaria was strongly associated with the level of formal education. Education remains a powerful tool that empowers people to enable them make decisions for themselves and influence their families [19].

The study revealed that majority (93.59\%) of participants had heard about malaria before. This is not surprising as malaria is a major public health problem in Ghana and the sub-region. The National Malaria Control Programme (NMCP), under the Advocacy, Communication and Social Mobilization (ACSM), Information Education and Communication (IE\&C) carried out behaviour change communication (BCC) activities during the year. The developed policy guidelines, manuals and a number of materials including data tools, printed and distributed to all regions [20]. Despite the high level of awareness about malaria mode of transmission (86\%) and methods of prevention (85\%), most of the participants had no LLIN. However a study found that the combination of IRS and LLIN provided significantly greater protection than the protection provided by ITN alone in preventing P. falciparum infection [21].

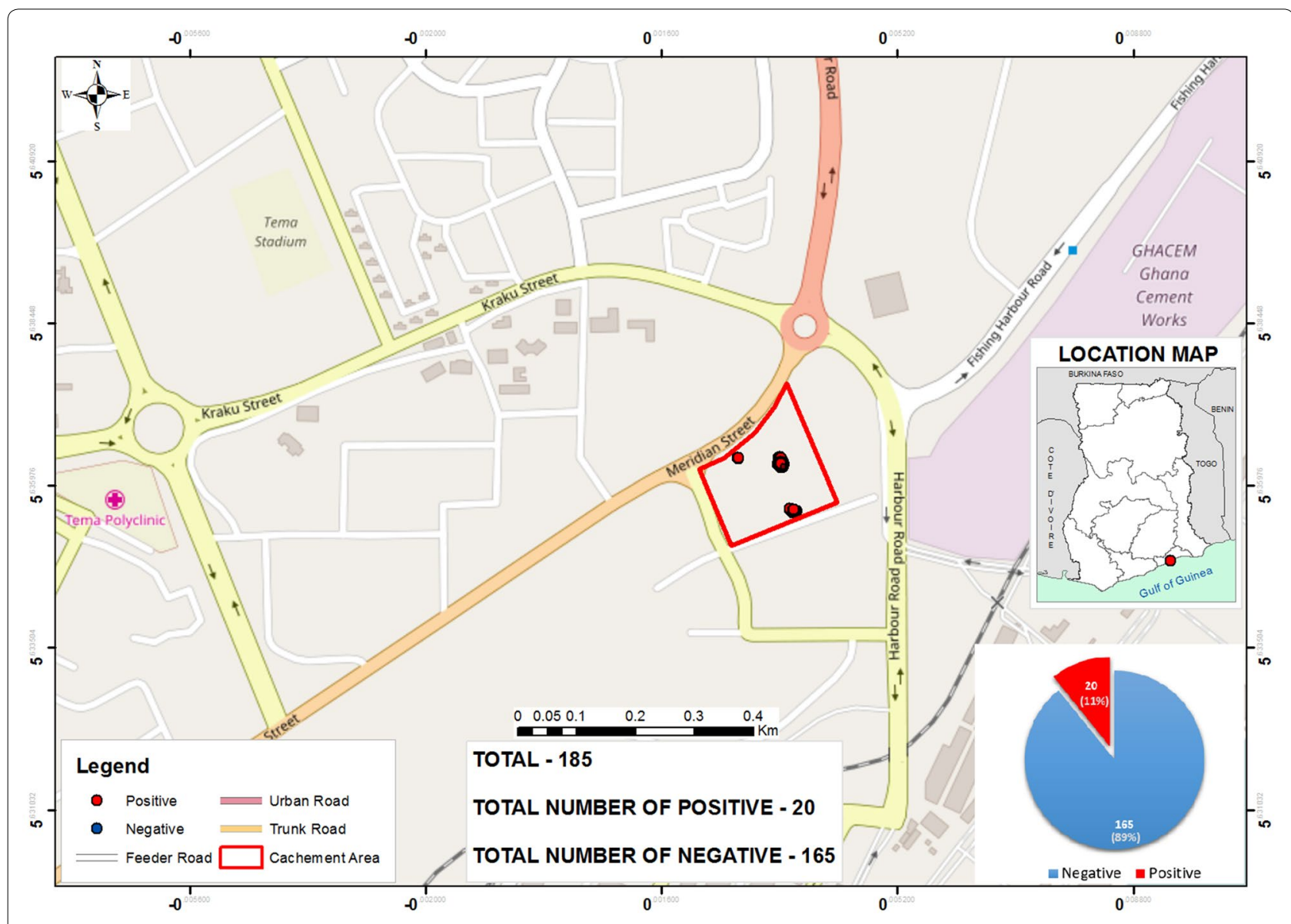

Fig. 3 Map showing Tema harbour and malaria prevalence 
Although mobile populations are generally described as hard-to-reach, this study used GPS technologies to identify a point of access to the target population where malaria control programmes could carry out interventions. Reports of malaria are increasing in many countries and in areas thought free of the disease. One of the factors contributing to the re-emergence of malaria is human migration. People move for a number of reasons, including environmental deterioration, economic necessity, conflicts, and natural disasters. These factors are most likely to affect the poor, many of whom live in the malaria-endemic countries. Identifying and understanding the influence of these population movements could improve prevention measures and malaria control programmes [8].

\section{Limitations}

The study had limitation in the sampling method used to recruit participants. Consecutive method was used because hawkers and long-distance truck drivers are hard to reach and do not have a census.

\section{Conclusion}

The prevalence of malaria is high among hawkers and long-distance truck drivers. The factors significantly associated with malaria in this population includes marital status, occupation and educational level. The GPS identifies the station locations associated with the prevalence of malaria in this mobile populations which will be useful in malaria interventions in Ghana.

\section{Authors' contributions \\ ND made contributions to conception, acquisition of data and analysis, and drafting of the manuscript. PA, EM and MA contributed to drafting and criti- cally reviewed the manuscript for intellectual content. BS conceived the study and designed it, and contributed to data analysis and manuscript writing and reviewing for intellectual content. All authors read and approved the final manuscript.}

\section{Author details}

${ }^{1}$ School of Public Health, University of Ghana, Legon-Accra, Accra, Ghana. ${ }^{2}$ Malaria Research and Training Center/Department of Epidemiology and Infectious Diseases/University of Science Techniques and Technologies of Bamako, Bamako, Mali.

\section{Acknowledgements \\ We are grateful to WHO/TDR, the Office of Research Innovation and Develop- ment of the University of Ghana and the National Malaria Control Programme for supporting this study. We extend our thanks to Francis Mike Pobee- Mensah, Ghana Ports and Harbours Authority, The Queen Mother of Madina market Women Association and all the study participants. We are also thankful to all the Research Assistants including Eric Mensah, Edward Dumashie," Juli- ana Marfo, Eric Adenankyage and Daniel Kiptoo who helped in collecting data from the study participants. Our special thanks go to Abubakari Bashara for all his supportive role in GIS mapping of the data.}

\section{Competing interests}

The authors declare that they have no competing interests.

\section{Availability of data and materials}

The datasets during and/or analysed during the current study available from the corresponding author on reasonable request.
Consent for publication

Participants' consents were obtained to publish this study.

\section{Ethical approval}

Ethical approval was obtained from the Ghana Health Service Ethical Review Committee (GHS-ERC 72/02/16).

\section{Funding}

This study received financial assistance from WHO/TDR. The funder had no role in study design, data collection and analysis, decision to publish, or preparation of the manuscript.

Received: 27 August 2016 Accepted: 25 February 2017

Published online: 09 March 2017

\section{References}

1. Snow RW. Global malaria eradication and the importance of Plasmodium falciparum epidemiology in Africa. BMC Med. 2015;13:23.

2. World Health Organization. World Malaria Report. Geneva: World Health Organization; 2016. http://www.who.int/malaria/publications/worldmalaria-report-2016/report/en/. Accessed on 30 Jan 2017.

3. World Health Organization. World malaria report. Geneva: World Health Organization; 2015. http://www.who.int/malaria/publications/worldmalaria-report-2015/report/en/. Accessed on 30 Jan 2017.

4. Smith C, Whittaker M. Beyond mobile populations: a critical review of the literature on malaria and population mobility and suggestions for future directions. Malar J. 2014;13:307.

5. Cohen JM, Smith DL, Cotter C, Ward A, Yamey G, Sabot OJ, et al. Malaria resurgence: a systematic review and assessment of its causes. Malar J. 2012;11:122.

6. Najera JA, Gonzalez-Silva M, Alonso PL. Some lessons for the future from the Global Malaria Eradication Programme (1955-1969). PLoS Med. 2011:8:e1000412.

7. Lynch C, Roper C. The transit phase of migration: circulation of malaria and its multidrug-resistant forms in Africa. PLoS Med. 2011;8:e1001040.

8. Martens P, Hall L. Malaria on the move: human population movement and malaria transmission. Emerg Infect Dis. 2000;6:103-9.

9. Wai KT, Kyaw MP, Oo T, Zaw P, Nyunt MH, Thida M, et al. Spatial distribution, work patterns, and perception towards malaria interventions among temporary mobile/migrant workers in artemisinin resistance containment zone. BMC Public Health. 2014;14:463.

10. Harris I, Sharrock WW, Bain LM, Gray K, Bobogare A, Boaz L, et al. A large proportion of asymptomatic Plasmodium infections with low and submicroscopic parasite densities in the low transmission setting of Temotu Province, Solomon Islands: challenges for malaria diagnostics in an elimination setting. Malar J. 2010:9:254.

11. Agyei-Mensah S, Aikins A. Epidemiological transition and the double burden of disease in Accra. Ghana J Urban Health. 2010;87:879-97.

12. National Malaria Control Programme. 2010 Annual Report, National Malaria Control Programme. Accra; 2010. http://www.ghanahealthservice.org/downloads/2010_Annual_Report_Final.pdf. Accessed Jan 2017.

13. Erhabor O, Azuonwu O, Frank-Peterside N. Malaria parasitaemia among long distance truck drivers in the Niger delta of Nigeria. Afr Health Sci. 2012;12:93-103.

14. Stanisic DI, Fowkes FJl, Koinari M, Javati S, Lin E, Kiniboro B, et al. Acquisition of antibodies against Plasmodium falciparum merozoites and malaria immunity in young children and the influence of age, force of infection, and magnitude of response. Infect Immun. 2015;83:646-60.

15. Ernst KC, Hayden MH, Olsen H, Cavanaugh JL, Ruberto I, Agawo M, et al. Comparing ownership and use of bed nets at two sites with differential malaria transmission in western Kenya. Malar J. 2016;15:217.

16. Schicker RS, Hiruy N, Melak B, Gelaye W. A venue-based survey of malaria, anemia and mobility patterns among migrant farm workers in Amhara Region, Ethiopia. PLoS ONE. 2015;10:e0143829.

17. Yegorov S, Galiwango RM, Ssemaganda A, Muwanga M, Wesonga I, Miiro $G$, et al. Low prevalence of laboratory-confirmed malaria in clinically diagnosed adult women from the Wakiso district of Uganda. Malar J. 2016;15:555. 
18. Okafor IP, Odeyemi KA. Use of insecticide-treated mosquito nets for children under five years in an urban area of Lagos State, Nigeria. Niger $\rfloor$ Clin Pract. 2012;15:220-3.

19. Kimbi HK, Nkesa SB, Ndamukong-nyanga JL, Sumbele IUN, Atashili J, Atanga MBS. Knowledge and perceptions towards malaria prevention among vulnerable groups in the Buea Health District, Cameroon. BMC Public Health. 2014;14:883.
20. National Malaria Control Programme. Annual Report National Malaria Control Programme. Accra; 2014. http://www.ghanahealthservice.org/ downloads/NMCP_2014_ANNUAL_REPORT.pdf.

21. Hamel MJ, Otieno P, Bayoh N, Kariuki S, Were V, Marwanga D, et al. The combination of indoor residual spraying and insecticide-treated nets provides added protection against malaria compared with insecticidetreated nets alone. Am J Trop Med Hyg. 2011;85:1080-6.

\section{Submit your next manuscript to BioMed Central and we will help you at every step:}

- We accept pre-submission inquiries

- Our selector tool helps you to find the most relevant journal

- We provide round the clock customer support

- Convenient online submission

- Thorough peer review

- Inclusion in PubMed and all major indexing services

- Maximum visibility for your research

Submit your manuscript at www.biomedcentral.com/submit 